\title{
Determination of the Resistance of Cone-Shaped Solid Electrodes
}

\author{
Frandsen, Henrik Lund; Hendriksen, Peter Vang; Koch, Søren; Kammer Hansen, Kent
}

Published in:

Journal of The Electrochemical Society

Link to article, DOI:

10.1149/2.0051711jes

Publication date:

2017

Document Version

Publisher's PDF, also known as Version of record

Link back to DTU Orbit

Citation (APA):

Frandsen, H. L., Hendriksen, P. V., Koch, S., \& Kammer Hansen, K. (2017). Determination of the Resistance of Cone-Shaped Solid Electrodes. Journal of The Electrochemical Society, 164(11), E3035-E3039.

https://doi.org/10.1149/2.0051711jes

\section{General rights}

Copyright and moral rights for the publications made accessible in the public portal are retained by the authors and/or other copyright owners and it is a condition of accessing publications that users recognise and abide by the legal requirements associated with these rights.

- Users may download and print one copy of any publication from the public portal for the purpose of private study or research.

- You may not further distribute the material or use it for any profit-making activity or commercial gain

- You may freely distribute the URL identifying the publication in the public portal

If you believe that this document breaches copyright please contact us providing details, and we will remove access to the work immediately and investigate your claim. 


\title{
Determination of the Resistance of Cone-Shaped Solid Electrodes
}

\author{
Henrik Lund Frandsen, ${ }^{\mathrm{Z}}$ Peter Vang Hendriksen, Søren Koch, and Kent Kammer Hansen
}

Department of Energy Conversion and Storage, Technical University of Denmark, DK-4000 Roskilde, Denmark

\begin{abstract}
A cone-shaped electrode pressed into an electrolyte can with advantage be utilized to characterize the electro-catalytic properties of the electrode, because it is less dependent on the electrode microstructure than e.g. thin porous composite electrodes, and reactions with the electrolyte occurring during processing can be avoided. Newman's formula for current constriction in the electrolyte is then used to deduce the active contact area based on the ohmic resistance of the cell, and from this the surface specific electro-catalytic activity. However, for electrode materials with low electrical conductivity (like $\mathrm{Ce}_{1-\mathrm{x}} \operatorname{Pr}_{\mathrm{x}} \mathrm{O}_{2-\delta}$ ), the resistance of the cell is significantly influenced by the ohmic resistance of the cone electrode, wherefore it must be included. In this work the ohmic resistance of a cone is modelled analytically based on simplified geometries. The two analytical models only differ by a model specific pre-factor, which is consequently determined by a finite element model. The model was applied to measurements on cones of $\mathrm{Ce}_{1-\mathrm{x}} \mathrm{Pr}_{\mathrm{x}} \mathrm{O}_{2-\delta} \mathrm{characterized}$ on an YSZ electrolyte. Conclusively, the finite element model was used to obtain a formula for the resistance for different cone angles with a small contact area. This reproduces Newman's formula for a cone angle equal to $90^{\circ}$, i.e. a semi-infinite body.

(C) The Author(s) 2017. Published by ECS. This is an open access article distributed under the terms of the Creative Commons Attribution Non-Commercial No Derivatives 4.0 License (CC BY-NC-ND, http://creativecommons.org/licenses/by-nc-nd/4.0/), which permits non-commercial reuse, distribution, and reproduction in any medium, provided the original work is not changed in any way and is properly cited. For permission for commercial reuse, please email: oa@electrochem.org. [DOI: 10.1149/2.0051711jes] All rights reserved.

(cc) BY-NC-ND
\end{abstract}

Manuscript submitted December 22, 2016; revised manuscript received February 24, 2017. Published March 11, 2017. This paper is part of the JES Focus Issue on Mathematical Modeling of Electrochemical Systems at Multiple Scales in Honor of John Newman.

Solid-state electrochemical devices find use in several different areas such as fuel cells, electrolyzers, sensors and cells for electrochemical flue gas purification. Most of these devices are still under development. One important task in this development is to be able to characterize electrodes in a simple, efficient and reliable way. Normally, the electrodes in solid-state chemistry are studied by the use of porous planar electrodes deposited on an electrolyte substrate, see e.g. ${ }^{1}$ This technique suffers the drawbacks that the performance of the electrode material is highly dependent on the electrode microstructure and a reaction between electrolyte and electrodes might occur during processing masking the "true" electrode performance. Another limitation in the use of planar porous electrodes is that electrode materials with a low electronic conductivity are difficult to characterize accurately as it, depending on the geometry, may be difficult to ensure that in-plane resistance and contact losses are negligible. Such electrode materials might, however still be highly relevant e.g. for use as infiltrate materials in an electrode backbone structure of good electronic conductivity due to their catalytic activity. ${ }^{2}$ It is therefore desirable to be able to evaluate the electro-catalytic properties of an electrode material independent of layer thicknesses and microstructural parameters.

An alternative to the use of porous planar electrodes is the use of cone-shaped electrodes. ${ }^{3}$ The use of cone-shaped electrodes in characterization of electrodes in solid-state chemistry is simple and less dependent on the electrode microstructure, and as the electrolyte and cone-shaped electrodes are fabricated separately, no reactions occur between the electrolyte and the electrode during component firing. In Figure 1 a sketch of the experimental setup for the utilization of cone shaped electrodes is shown. The current is induced at bottom of the electrolyte and collected at the top of the cone through platinum and gold paste, respectively. The cone electrode is located on top of the electrolyte, and in the interface between the two the electrochemical reaction occurs (depending on the available gas).

The purpose of this work is to enable deduction of the area specific electrocatalytic properties of the electrode material from the impedance characteristics, and from an estimate of the circular contact area, $A$. This area can be determined by modeling of the total ohmic resistance by including both the constriction resistance of the electrolyte and the resistance of the cone electrode. This is feasible as the ohmic resistance of the cone is highly sensitive to the contact area at the cone tip. In fact the resistance for small contact areas is only dependent on the diameter of the contact area and not the base diameter of the cone, as shown later. To obtain the contact diameter from the measured ohmic resistances presupposes that the conductivities of both the electrolyte and electrode are available. The ohmic resistance of the cone and electrolyte can be separated from the polarization resistances (from reactions, gas diffusion etc.) by impedance spectroscopy. The resistances reported onwards in this work are the ohmic resistances, which are obtained from impedance spectroscopy. Another requirement is that the polarization resistance of the working cone-shaped electrode has to be many times larger than the polarization resistance of the counter electrodes. This is achieved by having a counter electrode area many times larger than that of the tip of the cone-shaped electrode.

In the case of an electrode material with high electronic conductivity, the total ohmic resistance effectively equals that of the electrolyte as the ohmic resistance of the electrode can be neglected. The diameter of the circular contact area, $d$, can be calculated from the measured ohmic resistance with Newman's formula ${ }^{4}$

$$
d=\frac{1}{2 R_{\text {elyt }} \sigma_{\text {elyt }}}
$$

where $R_{\text {elyt }} \simeq R_{\text {total }}$ is the total ohmic resistance of the electrolyte. The conductivity of the electrolyte $\sigma_{\text {elyt }}$ needs to be known from literature or be measured in a separate experiment, e.g. by in-plane

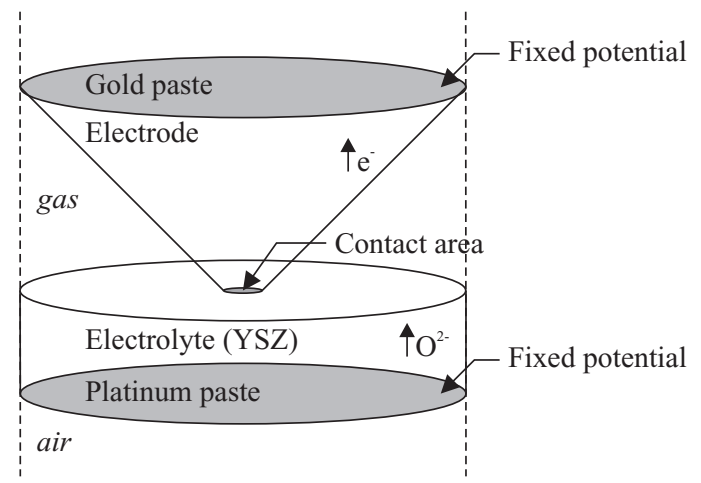

Figure 1. Conceptual sketch of the cone conductivity experimental setup. 
measurements. ${ }^{5}$ This expression applies to a circular area on a semiinfinite electrolyte. In practice it can be applied to an electrolyte, which has an extension bigger than $5 d .^{4}$

In the case of an electrode with low electronic conductivity, the total ohmic resistance found from impedance spectroscopy stems from both the electrolyte, $R_{\text {elyt }}$, and the electrode, $R_{\text {cone }}$

$$
R_{\text {total }}=R_{\text {cone }}+R_{\text {elyt }}
$$

Hence, the resistance of the cone must be expressed as a function of the contact diameter, after which a final expression for the contact area diameter can be established by solving of Eqs. 1 and 2 for the contact diameter.

In this work two analytical models for assessing the ohmic resistance of the electrode cone are derived. The form of the resulting expressions will be shown to be identical except for a model specific prefactor. The exact value of this factor was successively determined with finite element models of the cone electrode. The formulas for the resistance are of relevance for analysis of cone electrode measurements applied in solid electrochemistry, where the ohmic cone electrode resistance is not negligible. The resulting solution can also be utilized for any linear physical problem involving a cone shaped geometry which is governed by the Laplace equation (e.g. diffusion, heat transport, etc). Furthermore, the expression was generalized for arbitrary coneangles yielding a "generalized Newman's formula", which for a cone angle of $90^{\circ}$ replicates Newman's formula. The method was applied in analyzing measurements on cones of four different praseodymium doped cerium oxide cone-shaped electrodes $\mathrm{Ce}_{1-\mathrm{x}} \mathrm{Pr}_{\mathrm{x}} \mathrm{O}_{2-\delta}$ characterized on an YSZ electrolyte, published elsewhere. ${ }^{6}$ The purpose of this is to illustrate the significance of accounting for the resistance of the cone while analyzing the measurements.

\section{Analysis of the Cone Resistance}

In the following two analytical models for the electrical resistance of the cone and estimation of the contact area between a cone electrode and the electrolyte are derived. The models rely on a number of simplifying assumptions on the current flow and cone geometry. In both models it is assumed that there is a constant potential on the upper and lower boundary of the cone. This is a good approximation when all the interface area is active, all the electrochemical activity occurs at the interface between the electrode and the electrolyte, and the conductivity of the electrode and electrolyte are significantly different.

Stacked discs model.-In the first analytical model the resistance of the cone-shaped electrode is assessed by assuming that the cone can be described by a stack of horizontal thin circular plates with increasing diameter each with homogeneous vertical current field, see Figure $2 \mathrm{a}$. The resistance of each of the discs, $d R_{\text {cone }}$, can be calculated using Ohm's law:

$$
d R_{\text {cone }}=\frac{d z}{\sigma_{\text {cone }} A}=\frac{1}{\sigma_{\text {cone }}} \frac{1}{\pi r^{2}} d z
$$
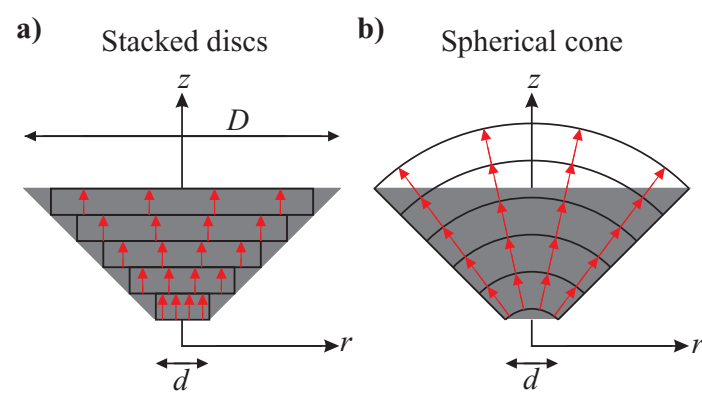

Figure 2. The 2 analytical model geometries and current flow field (shown with vectors): a) stacked discs b) spherical cone. where $d z$ is the thickness of the considered disc, $\sigma_{\text {cone }}$ is the specific conductivity of the cone electrode material, and $A$ is the area of the given plate. Here we consider a cone angle of $45^{\circ}$ (as in the experiments), and thus the radius of the plates equals the vertical coordinate $(r=z)$, see Figure 2a. The total resistance of the stack of electrode plates can then be obtained by integrating the resistances over the serially connected discs

$$
R_{\text {cone }}=\int_{d / 2}^{D / 2} d R_{\text {cone }}=\frac{1}{\pi \sigma_{\text {cone }}} \int_{d / 2}^{D / 2} \frac{1}{z^{2}} d z=\frac{4}{2 \pi \sigma_{\text {cone }}}\left(\frac{1}{d}-\frac{1}{D}\right)
$$

where $D$ is the base diameter of the cone, see Figure 2a.

The model specific factor $4 / \pi$ will for a simpler notification be denominated $Y$, such that the cone resistance is written as

$$
R_{\text {cone }}=\frac{Y}{2 \sigma_{\text {cone }}}\left(\frac{1}{d}-\frac{1}{D}\right)
$$

Inserting Eqs. 1 and 5 in Eq. 2 one gets

$$
R_{\text {total }}=\frac{1}{2 \sigma_{\text {elyt }} d}+\frac{Y}{2 \sigma_{\text {cone }}}\left(\frac{1}{d}-\frac{1}{D}\right)
$$

Isolating $d$ yields

$$
d=\frac{\frac{1}{2 \sigma_{\text {elyt }}}+\frac{Y}{2 \sigma_{\text {cone }}}}{R_{\text {total }}+\frac{Y}{2 \sigma_{\text {cone }}} \frac{1}{D}}
$$

As $D \gg d$ in the cone measurements $R_{\text {cone }}$ can be simplified to

$$
R_{\text {cone }}=\frac{Y}{2 \sigma_{\text {cone }}} \frac{1}{d}
$$

where $Y$ is thus seen to be the proportionality factor between the cone resistance and the inverse of the contact diameter. Eq. 7 may then be reduced to

$$
d=\left(\frac{1}{2 \sigma_{\text {elyt }}}+\frac{Y}{2 \sigma_{\text {cone }}}\right) \frac{1}{R_{\text {total }}}
$$

Spherical cone model.-In the second analytical model, the cone is assumed to be a conical section of a sphere, where the current is flowing radially from the contact zone, see Figure $2 b$. For a spherical cap, the curved area is:

$$
A=2 \pi r h
$$

where $h$ is the height of the cap, see Figure 3. In the case of a cone angle of $45^{\circ}, h$ can readily be deduced to be $(2-\sqrt{2}) r / 2$, see Figure 2b. Thus Eq. 10 becomes

$$
A=\pi(2-\sqrt{2}) r^{2}
$$

By assuming that the current runs perpendicular to the face of the cap, the resistance of a small part of the cone with thickness $d z$ becomes

$$
d R_{\text {cone }}=\frac{d z}{\sigma_{\text {cone }} A}=\frac{d z}{(2-\sqrt{2}) \pi \sigma_{\text {cone }} r^{2}}
$$




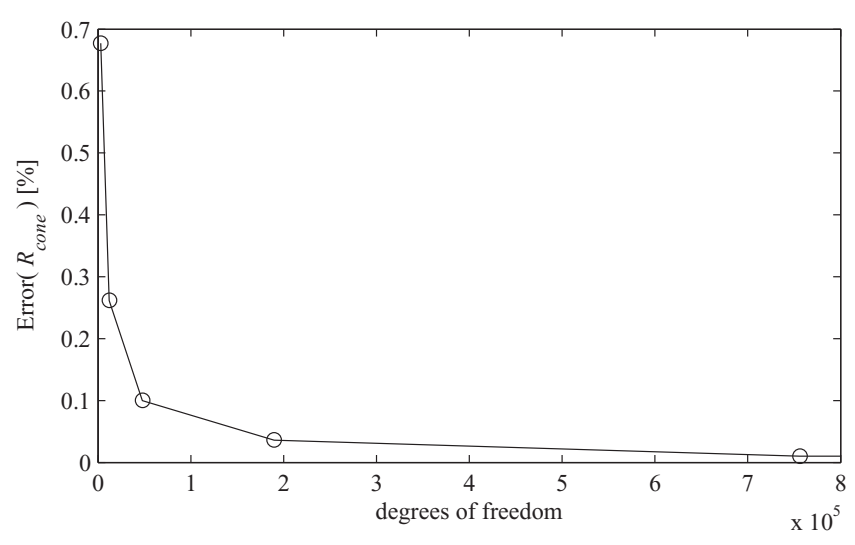

Figure 4. Error of the FEM as a function of degrees of freedom used.

The conical section starts at a distance $d / 2$ from origo and ends at a distance $D / 2$ as before, and the resistance of the cone is thus defined by the following integral

$$
\begin{aligned}
R_{\text {cone }} & =\int_{d / 2}^{D / 2} d R_{\text {cone }}=\frac{1}{(2-\sqrt{2}) \pi \sigma_{\text {cone }}} \int_{d / 2}^{D / 2} \frac{d z}{z^{2}} \\
& =\frac{4}{(2-\sqrt{2}) \pi 2 \sigma_{\text {cone }}}\left(\frac{1}{d}-\frac{1}{D}\right)
\end{aligned}
$$

This resembles Eq. 4 with the only difference that the proportionality factor $Y$ for this model is $4 /(2-\sqrt{2}) \pi$. Thus the Eqs. 5-9 applies for this model as well, however, with the proportionality factor $Y$ being equal to $4 /(2-\sqrt{2}) \pi$.

Finite element model.-The relationship between the cone resistance and the contact area diameter, $d$, is in Eq. 8 seen to be inversely proportional, and the resistance is independent of the base diameter of the cone, $D$, for $d \ll D$. The proportionality factor, $Y$, can also be determined exactly using a finite element model (FEM).

The FEM was built in the commercial software Comsol Multiphysics as an axis-symmetric model of the cone. From a convergence study it was observed that by using approximately $8 \cdot 10^{5}$ of degrees of freedom the cone resistance could be determined with less than $0.1 \%$ percentage of error, see Figure 4. Doing so the proportionality factor $Y$ was determined to be 1.732 with a goodness of fit $R^{2}$ equal to 1.000 .

\section{Experimental}

Four different compositions of $\mathrm{Ce}_{1-\mathrm{x}} \mathrm{Pr}_{\mathrm{x}} \mathrm{O}_{2-\delta}(\mathrm{CPO})$ have been prepared (with $\mathrm{x}$ equal to 10, 10, 30 and 40). Further details of the preparation of the electrodes material compositions is described elsewhere. ${ }^{6}$

The electrode cones were machined to have a cone angle of $45^{\circ}$, and a base diameter of $7.5 \mathrm{~mm}$. The electrochemical impedance spectroscopy was recorded at $500^{\circ} \mathrm{C}$ in an atmosphere of $10 \%$ oxygen in argon. The impedance was recorded from $30 \mathrm{kHz}$ to $50 \mathrm{mHz}$ with 6 points per decade and an amplitude of $25 \mathrm{mV}$ RMS (root mean square) at open circuit voltage. For the measurements a Gamry Femtostat was used. As an electrolyte yttria stabilized zirconia was used. The conductivity of the YSZ electrolyte was assumed to be $1.42 \cdot 10^{-3} \mathrm{Scm}^{-1}$ at $500^{\circ} \mathrm{C}^{7}$

The series resistance found from impedance spectroscopy is given in the Table I, together with the specific conductivity of the four CPO material compositions determined by four point DC conductivity measurements at $500^{\circ} \mathrm{C}$.

In praxis we are measuring on a electrode multi-contact point for which gas diffusion into the interface between electrode and electrolyte is not a limitation, and the measured resistance is thus mainly

\begin{abstract}
Table I. Conductivity of the electrode material $\mathrm{Ce}_{1-x} \operatorname{Pr}_{x} \mathrm{O}_{2-\delta}$ with different degrees of substitution of praseodymium, $x$ and corresponding measured total resistances. The $\mathbf{R}_{\text {cone }} / \mathbf{R}_{\text {elyt }}$ ratio is estimated with $Y$ determined by the FEM.
\end{abstract}

\begin{tabular}{ccccc} 
Name & Material & $\mathrm{R}_{\text {total }}[\Omega]$ & $\begin{array}{c}\text { Conductivity } \\
{\left[\mathrm{Scm}^{-1}\right]^{9}}\end{array}$ & $\mathrm{R}_{\text {cone }} / \mathrm{R}_{\text {elyt }}$ \\
\hline CPO10 & $\mathrm{Ce}_{0.9} \operatorname{Pr}_{0.1} \mathrm{O}_{2-\delta}$ & $5.10 \cdot 10^{5}$ & $3.20 \cdot 10^{-4}$ & 7.7 \\
$\mathrm{CPO} 20$ & $\mathrm{Ce}_{0.8} \operatorname{Pr}_{0.2} \mathrm{O}_{2-\delta}$ & $3.60 \cdot 10^{5}$ & $1.30 \cdot 10^{-3}$ & 1.9 \\
$\mathrm{CPO} 30$ & $\mathrm{Ce}_{0.7} \operatorname{Pr}_{0.3} \mathrm{O}_{2-\delta}$ & $4.10 \cdot 10^{5}$ & $3.20 \cdot 10^{-3}$ & 0.8 \\
CPO40 & $\mathrm{Ce}_{0.6} \operatorname{Pr}_{0.4} \mathrm{O}_{2-\delta}$ & $5.90 \cdot 10^{5}$ & $1.30 \cdot 10^{-2}$ & 0.2
\end{tabular}

due to the electronic charge transport in the electrode material and polarization resistance from the electrode reaction occurring in the interface. ${ }^{8}$ Thus, here it is assumed that electrode reaction occurs over the entire interface area, i.e. that gas access occurs via small porosities, and that the gas is distributed fast to ensuring an even distribution of the reactions across the interface.

\section{Results and Discussion}

Comparison of numerical and analytical models.-In Table II the proportionality factors between the cone resistance and the contact diameter from the different methods are presented. The correct proportionality factor obtained by FEM is seen to be very close to the average of the two proportionality factors obtained by analytical methods ( $0.5 \%$ deviation).

The first analytical method (stacked discs) represents the case, where the correct shape of the contact area is modeled, but the current lines are "discontinuous" as only the vertical movement of current is accounted for and the horizontal movement is neglected (see Figure 2a). This approach will estimate a too low proportionality factor (1.273), since the horizontal movement of charge occurs without any resistance (only vertical movement of charge is accounted for) as shown in Figure 2a.

The second method (spherical cone) represents a better model of the current lines as the current lines distributes radially as seen in the FEM, see Figure 5, but the geometry of the contact area is not correctly represented.

In Table III the calculated (by Eq. 7 and Eq. 9) contact diameters for the cones of different CPO compositions are shown. Notice that the error of the analytical methods increases with increasing contact diameter, also relatively. In the estimation of the contact diameter the two analytical methods under and overestimates the contact area, respectively.

Size dependency.-As mentioned above the solutions for the analytical models can be simplified (Eq. 8) for geometries relevant to the experimental conditions, i.e. a contact diameter much smaller than the base diameter of the cone $(d \ll D)$. To know the applicability of Eq. 8 , the accuracy of it is determined for different cone sizes.

As the differential equation for charge transfer is linear for constant conductivity, a scalable solution can be obtained for different geometries with the same aspect ratio, $\delta=D / d$. Thus, the resistance of the cone will scale linearly with the size of it, and the size dependency investigation can be reduced to depend on only one length parameter, which is chosen to be $\delta=D / d$.

Table II. Proportionality factor between cone resistance and contact diameter from the different methods.

\begin{tabular}{cccc} 
Model & Stack of discs & Spherical cone & FEM \\
\hline$Y$ & $\frac{4}{\pi}$ & $\frac{4}{(2-\sqrt{2}) \pi}$ & \\
$\operatorname{num}(Y)$ & 1.273 & 2.174 & 1.732
\end{tabular}




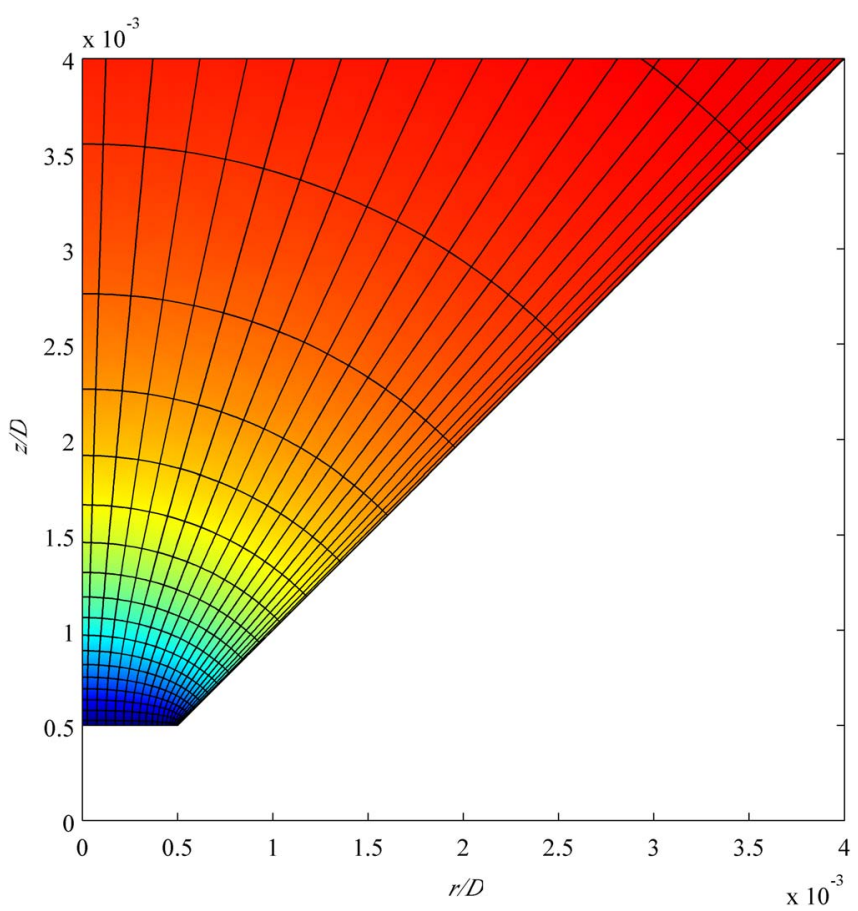

Figure 5. Current lines (vertical curved) and equipotential lines (horizontal curved) in the vicinity of the contact area obtained by the FEM.

In Figure 6 the variation of the scaling factor $Y$ with $\delta$ is shown for the three different models, i.e. the stacked disc, spherical cone and FEM together with the average of the two analytical models. The dependency of $Y$ in the analytical models is given by Eq. 5 and the simplified version in Eq. 8 (marked with asterisk, ${ }^{*}$ ). For all models a constant cone angle of $45^{\circ}$ is set.

The resistance of the cone can then be expressed in terms of a dimensionless proportionality parameter, $Y_{f}$, which is here defined as

$$
Y_{f}(\delta) \equiv R_{\text {cone }} 2 \sigma_{\text {cone }} d
$$

such that $Y_{f}$ tends toward $Y$ for $\delta$ going toward infinity, see Eq. 8 .

Although the average of the two analytical solutions is equal to that of the FEM for larger aspect ratios (Eq. 8), they are seen to produce unlike trends for smaller aspect ratios (described in Eq. 5). However, even for $\delta>100$ less than $1 \%$ of error is committed by assuming that $Y_{f}=1.732$, see Figure 7 .

Cone angle dependency.-Another finite element model was made to determine $Y$ for various cone angles, whereas the above analytical solutions only apply for a cone angle of $45^{\circ}$. In the current FEM the far field was represented by a cone section of a sphere, see Figure 8, where the diameter of the sphere was a 1000 times larger than the contact diameter. Hereby the influence of the far field becomes negligible.

Table III. Contact diameters determined for the different electrode compositions for the direct formulation, Eq. 7 and the approximated expression Eq. 9 marked by asterisk $\left({ }^{*}\right)$.

\begin{tabular}{lccccc} 
& \multicolumn{5}{c}{ Contact diameter $[\mu \mathrm{m}]$} \\
\cline { 2 - 6 } Name & $\begin{array}{c}\text { Stack of } \\
\text { discs }\end{array}$ & $\begin{array}{c}\text { Stack of } \\
\text { discs* }\end{array}$ & $\begin{array}{c}\text { Spher. } \\
\text { cone }\end{array}$ & $\begin{array}{c}\text { Spher. } \\
\text { Cone* }^{*}\end{array}$ & FEM \\
\hline CPO10 & 45.9 & 45.6 & 73.5 & 72.5 & 60.0 \\
CPO20 & 23.4 & 23.3 & 33.0 & 32.9 & 28.3 \\
CPO30 & 13.4 & 13.4 & 16.9 & 16.8 & 15.2 \\
CPO40 & 6.8 & 6.8 & 7.4 & 7.4 & 7.1
\end{tabular}

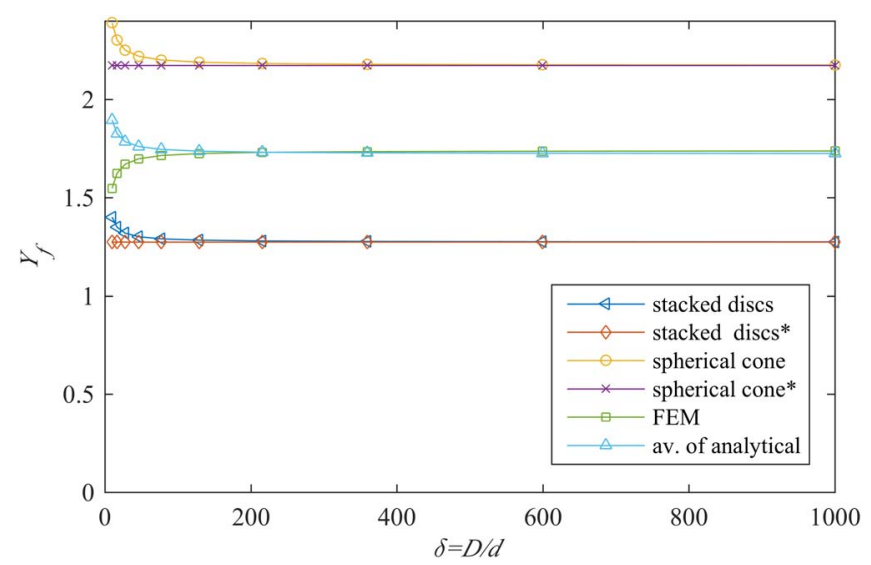

Figure 6. Variation of $Y$ with increasing aspect ratio, $\delta$, for the different models. The asterisk $\left({ }^{*}\right)$ indicates the approximate solution in Eq. 8.

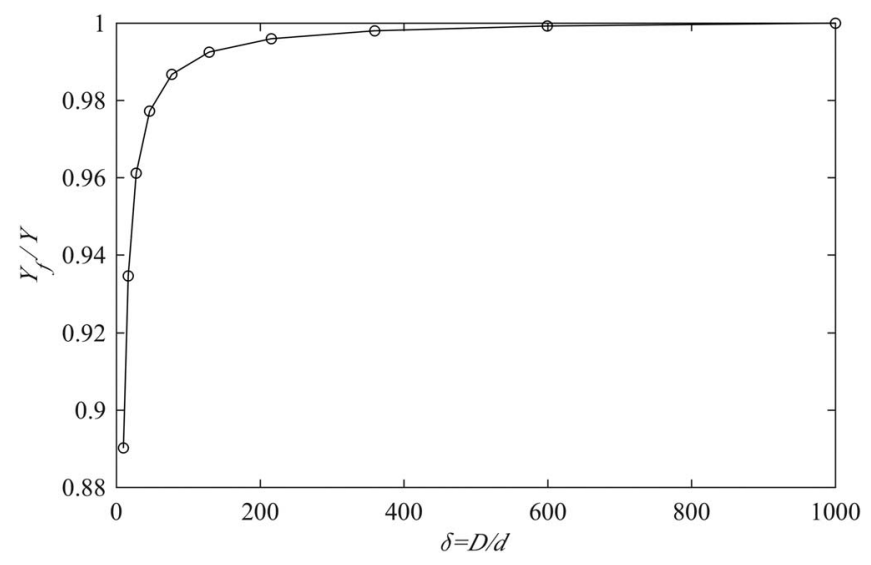

Figure 7. Variation of $Y / Y_{\infty}$ with increasing aspect ratio, $\delta$, in the FEM.

For a cone angle, $\theta$, of $90^{\circ}$ this model provides Newman's formula (Eq. 1), with a small deviation (1\%), which is due to numerical errors, see Figure 9. In Figure 9 the variation of $Y$ for different cone angles is shown together with a fit of the FEM results with the formula

$$
Y=\left(\frac{a+\theta}{a+90^{\circ}}\right)^{b}
$$

where $a$ and $b$ are fitting constants. $a$ and $b$ were obtained by least square fitting of Eq. 15 to the FEM calculated values of $Y$ for the cone angle, $\theta$, ranging from $30^{\circ}$ to $90^{\circ}$ to be $-9.278^{\circ}$ and -0.6797 , respectively, with a goodness of fit $\mathrm{R}^{2}=0.9998$. To achieve a good fit the range of cone angles was chosen to experimentally realistic ones. For smaller cone angles the resistance goes to infinity (for an infinitely long cylinder), and including these values in the fitting results in an inaccurate fit for the larger more realistic cone angles. The values of $Y$ are also shown in Table IV.

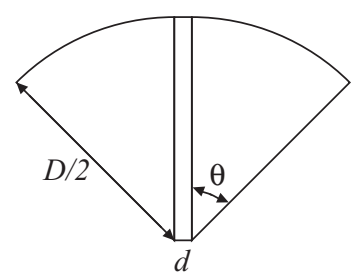

Figure 8. Sketch of geometry of the FEM for varying cone angles, $\theta$. 
Table IV. Variation of $Y_{f}$ with cone angles, $\theta$, obtained by FEM.

\begin{tabular}{ccccccccccccc}
$\theta\left[^{\circ}\right]$ & 35 & 40 & 45 & 50 & 55 & 60 & 65 & 70 & 75 & 80 & 85 & 90 \\
\hline$Y_{f}$ & 2.182 & 1.933 & 1.742 & 1.590 & 1.467 & 1.367 & 1.282 & 1.211 & 1.150 & 1.098 & 1.053 & 1.013
\end{tabular}

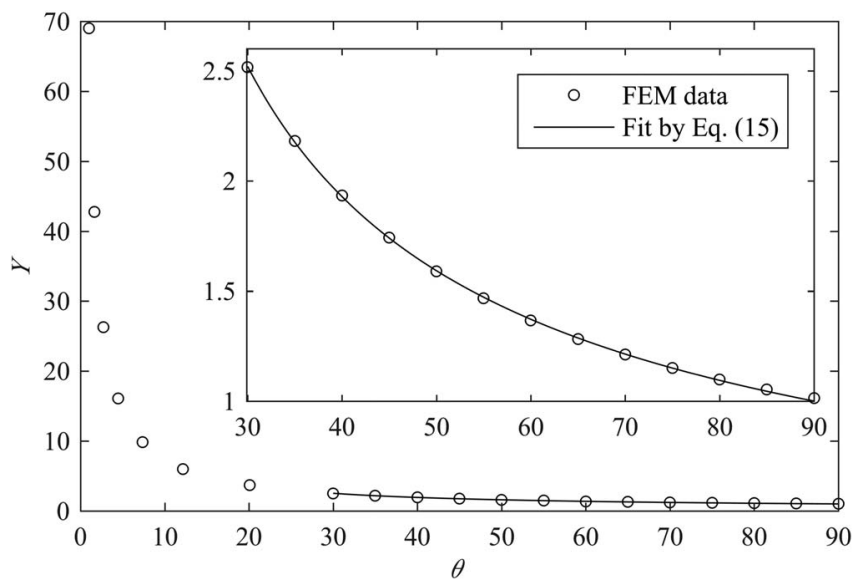

Figure 9. Variation of $Y$ with cone angles, $\theta$, obtained by FEM and fitted with Eq. 15.

Thus, Eq. 8 together with Eq. 15 yields a generalized Newman's formula, which can be utilized for arbitrary cone angles.

$$
R_{\text {cone }}=\left(\frac{a+\theta}{a+90^{\circ}}\right)^{b} \frac{1}{2 \sigma_{\text {cone }}} \frac{1}{d}
$$

\section{Conclusions}

In this paper a model to assess the ohmic resistance of a coneshaped electrode has been presented. This is of relevance for correct data treatment of cone electrode studies, where the ohmic losses in the cone itself are not negligible.

First two analytical models based on geometric simplifications, i.e. a series of stacked discs and a spherical cone, were derived. The form of these two models suggested that the resistance of the cone could be expressed as a model specific factor over the conductance of the cone material and the contact area diameter. The model specific factors of the two models were however unlike, and therefore the exact value of the parameter was consequently determined by finite element modeling. The model specific factor of the finite element model was found to be close to the average of those of the two analytical models ( $0.5 \%$ deviation).

The requirement for the analytical model simplification was however that the contact diameter should be much smaller than the base diameter of the cone. To investigate the range of validity of this assumption the error committed for smaller diameters were studied by use of the finite element model. It was found that for a ratio of the base diameter to the contact diameter higher than 100, the resistance could be determined with an error of less than $1 \%$.

The resulting models were applied to measurements on cones of different compositions of $\mathrm{Ce}_{1-\mathrm{x}} \mathrm{Pr}_{\mathrm{x}} \mathrm{O}_{2-\delta}$.

The resistances of cones with arbitrary angles and a small contact area were also determined by several finite element models. Fitting a curve to these results in a general equation for the resistance of a cone for varying cone angles. For a cone angle of $90^{\circ}$, the equation reproduces Newman's formula for current constriction in a semiinfinite body.

\section{Acknowledgments}

The work has been founded by The Danish Council for Strategic Research (project \#09-065186). The financial support is gratefully acknowledged.

\section{References}

1. M. Juhl, S. Primdahl, C. Manon, and M. Mogensen, J.Power Sources., 61, 1 (1996).

2. D. Ippolito and K. K. Hansen, Journal of Solid State Electrochemistry., 17, 3 (2013).

3. P. Fabry and M. Kleitz, J Electroanal Chem., 57, 2 (1974).

4. J. Newman, J.Electrochem.Soc., 113, 5 (1966).

5. I. Riess and D. S. Tannhauser, Solid State Ionics., 7, 4 (1982).

6. R. M. L. Werchmeister and K. K. Hansen, Electrochim. Acta. (Accepted).

7. C. C. Appel, N. Bonanos, A. Horsewell, and S. Linderoth, J.Mater.Sci., 36, 18 (2001)

8. P. Blennow, K. K. Hansen, L. R. Wallenberg, and M. Mogensen, Electrochim.Acta., 52, 4 (2006)

9. Y. Takasu, T. Sugino, and Y. Matsuda, J.Appl.Electrochem., 14, 1 (1984). 